\title{
Patterns of granular convection and separation in narrow vibration bed
}

\author{
Chuanping Liu, ${ }^{1, *}$, Ping $\mathrm{Wu}^{2}$, Li Wang ${ }^{1}$, Lige Tong ${ }^{2}$, Shaowu Yin ${ }^{1}$ \\ ${ }^{1}$ School of Energy and Environment Engineering, University of Science and Technology Beijing, 100083, Beijing, China \\ ${ }^{2}$ School of Mathematics and Physics, University of Science and Technology Beijing, 100083, Beijing, China \\ ${ }^{3}$ Beijing Engineering Research Centre of Energy Saving and Environmental Protection, 100083, Beijing, China
}

\begin{abstract}
Granular convection/separation of single and binary component particles are studied in a narrow vibration bed, respectively. With filling the single light particles (molecular sieve beads), the bed exhibits five different states successively by increasing the vibration frequency $\mathrm{f}$ from $15 \mathrm{~Hz}$ to $70 \mathrm{~Hz}$ (vibration strength $\Gamma>3$ ), as the global convection, symmetrical heap, unsymmetrical heap, local convection and pseudo solid. Comparatively, the granular bed of the single heavy particles (steel beads) is only in pseudo solid state at the above vibration condition. By filling binary component particles (molecular sieve and same size steel beads) instead of the single component, the bed shows similar convection state with that of the single molecular sieve beads, and the heavy steel beads are aggregated in the centre of convention roll as a core. Varying the initial distribution of binary component particles, the final convection and separation are not influenced, although the aggregation process of steel beads changes.
\end{abstract}

\section{Introduction}

Granular matter wildly exist in industry and agriculture. Due to the complex force chain structure among particles and the dissipation of kinetic energy during inelastic collision, granular matter usually exhibits interesting behaviors different with traditional liquid and solid, such as convection [1,2] and separation $[3,4]$.

Under externally vibration, binary mixture would show diffrent types of separation. Size segregation is known as the Brazil nut (BN) effect when large particles rise to the top; by contrast, in the reverse effect, called the reverse $\mathrm{BN}$ (RBN) effect, large particles sink to the bottom. Separation may appear in particle species with different density besides different size. King et al. [5,6] investigated equal-sized glass-copper system and found that heavy copper powder rises to the bed top, whereas light glass powder sinks to the bed bottom at low vibration frequencies. However, at high vibration frequencies, the mixture separates into the "Sandwich" configuration, with copper powders distributed at the middle of the bed. Shi et al. [7] found that light particles tend to rise and form a pure layer on the top of the granular bed, with a mixed part of heavy particles and some light particles at the bottom of the bed; these particles form a partially separation state. Besides "BN", "RBN" and "Sandwich" separation, several studies [8-11] reported that clusters can be formed in the granular bed.

Convection is a representative mechanism used to explain granular separation [4]. With the upward stream of granular convection, the large particles rise to bed top; however, they cannot sink back with the downward stream, as the large particles size is larger than the convection zone width of the downward stream, resulting in "BN" separation. Only mild convection rates result in size separation because strong convections can enhance mixing [12]. Yang et al. [9] indicated that two convection cells exist in a vibrated system, and heavy particles concentrate near the cell centers. Hsiau [11] investigated the effect of container geometry on density separation. At the few filling layers, heavier particles migrate to the bottom of the bed. For the high filling layers, the bulk convection motion is the main mechanism that affects separation; in this case, heavier particles accumulate in the center of convection.

Ehrichs et al. [13] measured the velocity distribution of the particles in cylinder vibration bed by NMR. The particles move upward in the middle of the bed and downward along the wall, resulting a pair of symmetric convection. In the experiments of Wong et al. [14], for the frequency range from $14 \mathrm{~Hz}$ to $18 \mathrm{~Hz}$, an unsymmetrical convection roll with the formation of heap against one of the sidewall was observed. And Akiyama's research [15] indicated that the interstitial gas plays a non-negligible role on granular heap. According to the simulation performed by Majid [8], vibration parameters and container geometries also are the important factors effecting the granular convection. Eshuis [16] and Zhang [17] observed multiple patterns of granular motion and presented their phase diagram by varying the vibration amplitude and frequency.

Serval literatures $[4,9,11]$ indicated that the separation, especilly density separation, is induced by

* Corresponding author: cpliu@ustb.edu.cn 
granular convection. This paper aimed to further study the granular convection and separation under vertical vibration, and discuss the mutual relations between convection and separation. Firstly, the single component particles $[13 \mathrm{X}$ molecular sieve (13XMS, sintered aluminosilicate adsorbing material) / steel beads] are filled in vibration bed, and different types of convection patterns are observed at our experimental condition, which are influenced by both the vibration parameters, especially vibration frequency. Secondly, the 13XMS and the same size steel beads are initially layered in bed. The convection patterns and separation process of binary component particles are analyzed by comparing with that of single component.

\section{Experimental setting}

The following experiments are performed in a narrow rectangle glass bed of size $200 \mathrm{~mm}$ (length) $\times 5$ $\mathrm{mm}$ (width) $\times 220 \mathrm{~mm}$ (height). As shown in Figure 1, the bed is fixed at a vibration platform of a Brüel \& Kjær standard vibrating system (LDS V555), which can generate sinusoidal vibration in the form of $z=A \sin (2 \pi f t)$, where $A$ and $f$ are the vibration amplitude and frequency, respectively. The experimental particles include spherical 13XMS and steel beads. The physical properties of the two kinds of particles are listed in Table 1. Bulk density $\left(\rho_{b}\right)$ is the ratio of the total mass of single particle piles to the volume that these particles occupy, including the voids. A charge-coupled device (CCD) camera was fixed at the front of the bed to record particle motions in vibration bed.

The single component or the binary component particles are initially filled into glass bed with a certain granular layer height $(h)$. In our experiments, vibration frequency $(f)$ is adjusted from $20 \mathrm{~Hz}$ to $70 \mathrm{~Hz}$, and dimensionless vibration acceleration $(\Gamma)$ from 0 to 8 , where, $\Gamma=A(2 \pi f)^{2} / g$ and $g$ is gravitational acceleration. We recorded the particle motion at the bed front by using a CCD camera. Basing on the continuous images from the $200 \mathrm{fps}$ CCD recordings, we got the movement of the particles in vibration bed.

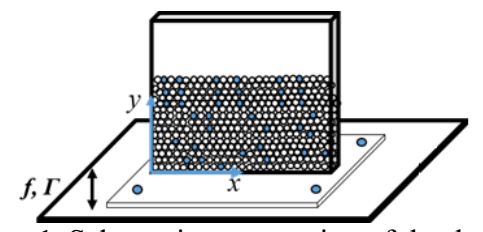

Figure 1. Schematic presentation of the device.

Table 1. Physical properties of the two kinds of particles selected in our experiment.

\begin{tabular}{ccccc}
\hline Material & $\begin{array}{c}\text { Diameter } \\
d(\mathrm{~mm})\end{array}$ & $\begin{array}{c}\text { Material density } \\
\rho_{m}\left(\mathrm{~g} / \mathrm{cm}^{3}\right)\end{array}$ & $\begin{array}{c}\text { Bulk density } \\
\rho_{b}\left(\mathrm{~g} / \mathrm{cm}^{3}\right)\end{array}$ & $\begin{array}{c}\text { Sphericity } \\
\Phi\end{array}$ \\
\hline 13XMS & $2.00 \pm 0.10$ & $1.47 \pm 0.08$ & $0.82 \pm 0.08$ & 0.92 \\
Steel beads & $2.00 \pm 0.05$ & $8.60 \pm 0.05$ & $4.82 \pm 0.26$ & 0.96 \\
\hline
\end{tabular}

\section{Experimental results}

\subsection{Convection of single component}

We filled the 13XMS beads into the bed with 160 $\mathrm{mm}$ height $(h)$, and then vibrated it at $\Gamma=4.0$. The granular bed exhibits five different states by increasing the vibration frequency gradually from $15 \mathrm{~Hz}$ to $70 \mathrm{~Hz}$ [Figure 2(I) (VI)].

(1) Global convection. When the vibration frequency $f<25 \mathrm{~Hz}$, the whole granular bed is completely fluidized under large amplitude vibration. As shown in Figure 2(I), The particles at the middle of the bed rise to the bed top quickly. At the bed surface, particles bound from bed center to sidewall and then sink along the bed wall. The whole bed shows one pair of symmetrical strong global convection roll.

(2) Symmetrical heap. By increasing frequency $(25$ $\mathrm{Hz}<f<34 \mathrm{~Hz}$ ), the vibration amplitude decreases and the particles in bed are cooled. Comparing with the global convection, although the granular convection always exists, its strength weakens obviously. The bottom center of the granular bed starts to be solidified, that is, the particles in the bottom center vibrate in place and cannot migrate to another position. Meanwhile, a symmetrical granular heap occurs at the bed top. The convective particles rise from the bed middle to the heap peak, and then flow downward at the heap surface, as shown in Figure 2(II).

(3) Unsymmetrical heap. When the frequency increases further $(34 \mathrm{~Hz}<f<47 \mathrm{~Hz})$, the solidified zone extends to the whole bed bottom and only the particles at the upper bed take part in convection. The original unsymmetrical form of the heap is broken, resulting in one pair of unsymmetrical convection roll with the rising point deviating from the center of the bed, as shown in Figure 2(III). One roll extends over a larger volume of the bed than the other, and the convection strength of the larger rolls is higher than that of the smaller one.

(4) Local convection. When the frequency $47 \mathrm{~Hz}<f$ $<66 \mathrm{~Hz}$, most of the bed is solidified except the bed top near the wall. The granular convection only occur at the top corner of the bed and the bed surface re-becomes flat, as shown in Figure 2(IV).

(5) Pseudo solid. When the frequency $f>66 \mathrm{~Hz}$, the whole bed is almost solidified. All the particles vibrate in place and the bed surface about keeps flat, as shown in Figure 2(V).

By varying both the vibration frequency $f$ and acceleration $\Gamma$, we can got the phase diagram of the above five patterns of granular motion, as shown Figure 3(a). When $\Gamma<1.2$, the granular bed is in pseudo solid state as the vibration strength is too weak. When $\Gamma>3$, the patterns are mainly influenced by the vibration frequency, and the vibration acceleration is a minor 
factor. The $\Gamma$ only less affects the $f$ range of each granular motion pattern.

By filling the steel beads into the bed with $h=160$ $\mathrm{mm}$, and vibrating the bed at $\Gamma=4.0$, the granular bed only exhibits Pseudo solid state during the frequency 15 $\mathrm{Hz}<f<70 \mathrm{~Hz}$. The bed surface keeps flat, as shown in Figure2(VI), being same with Figure 2(V). As the steel beads are much heavier than 13XMS, the bed filled heavy steel beads cannot be fluidized at same vibration condition. The steel beads vibration together and keep relative still each other. Changing the vibration parameter, the steel beads hardly generate any convection during $15 \mathrm{~Hz}<f<70 \mathrm{~Hz}$ and $0<\Gamma<8$. The phase diagram of steel beads only has the pseudo solid state, as shown in Figure 3(b).

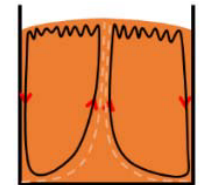

(I)

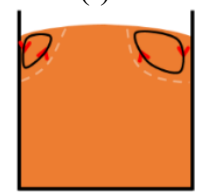

(IV)

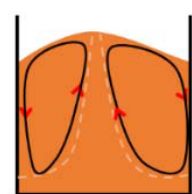

(II)

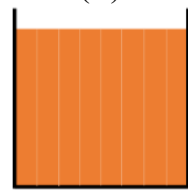

(V)

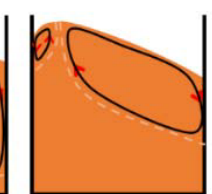

(III)

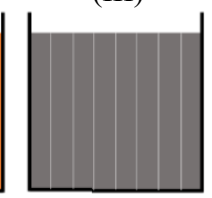

(VI)
Figure 2. Different types of granular motion $[(\mathrm{I}) \sim(\mathrm{V})$ for $13 \mathrm{XMS}$ and (VI) for steel beads]
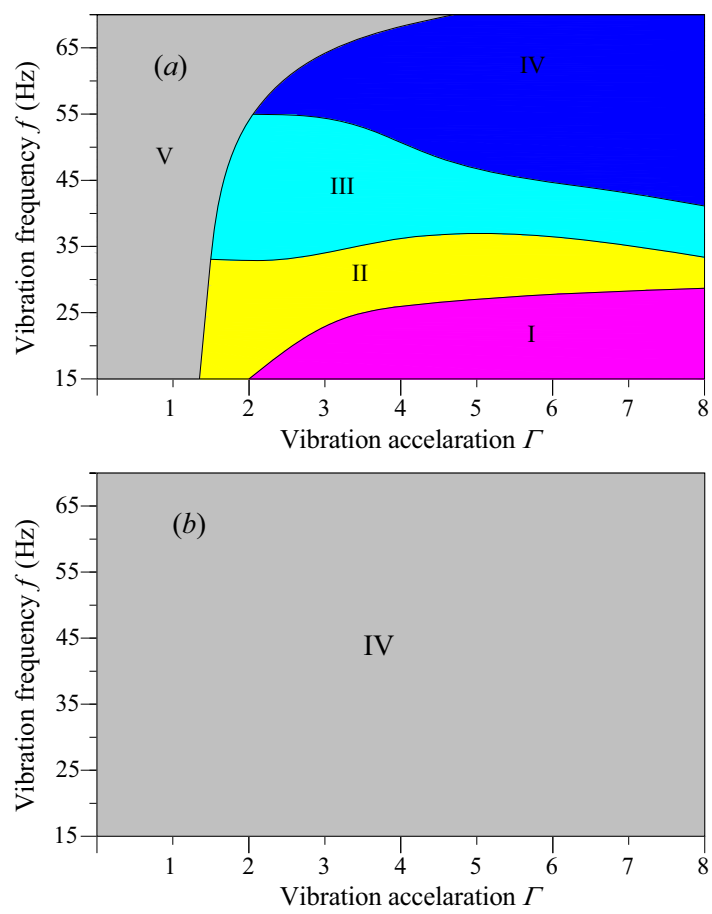

Figure 3. Phase diagram of granular motion [(a) for 13XMS and (b) for steel beads]

\subsection{Convection and separation of binary component}

We filled the 13XMS beads into the bed with 144 $\mathrm{mm}$ height $(h)$ and then covered $16 \mathrm{~mm}$ height steel beads layer on the 13XMS [Figure 4(a1)]. Once vibrating the bed at $\Gamma=4.0$ and $f=60 \mathrm{~Hz}$, the granular convection occurs, being similar with Figure 2(IV). The steel beads on the bed top gradually move toward sidewall and aggregate at the top corner [Figure 4(a2)]. The 13XMS beads move upward from the bed center, then over the steel beads layer [Figure 4(a3)]. As the $13 \mathrm{XMS}$ beads cover on steel beads and do convective motion, the steel beads are wrapped in the 13XMS. After the vibration starts $5 \mathrm{~min}$, the bed exhibits a stable distribution, as shown in Figure 4(a4), Most of the heavy steel beads cluster in the center of the convection, as a core of convection roll.

The $16 \mathrm{~mm}$ steel beads layer was initially placed below the 13XMS beads [Figure 4(b1)]. By vibrating the bed at $\Gamma=4.0$ and $f=60 \mathrm{~Hz}$, we recorded the steel beads movement. The steel beads at the bottom firstly rise slowly and move through the 13XMS bed [Figure4 (b1) (b4)]. After about 10min, they reach the bed surface, as Figure 4(b5). Then the steel beads move toward sidewall and finally format a convection core [Figure 4(b6)].

By varying the vibration frequency, the granular convection and separation of the 13XMS beads also change. Figure 5 (a) $\sim(d)$ show the stable convection and separation at $f=20 \mathrm{~Hz}, 30 \mathrm{~Hz}, 40 \mathrm{~Hz}$ and $60 \mathrm{~Hz}$. As the volume ratio of steel beads is much less than that of 13XMS, the effect of steel beads have hardly effect on granular convection, which is almost same with that of single 13XMS bead [Figure 2(I) (IV)]. In the solidified zone, there are few steel beads. The steel beads are wrapped in the granular convection roll as a granular core. The much strong the convection is, the looser the core (steel beads) is.

We filled the steel beads into the bed with $144 \mathrm{~mm}$ height $(h)$ and then covered $16 \mathrm{~mm}$ height 13XMS layer on the steel beads [Figure 6(a1)]. The bed was vibrated at $\Gamma=4.0$ and $f=60 \mathrm{~Hz}$. At the beginning, the steel beads are in solidified state, and the 13XMS beads at the bed surface move toward sidewall, then downward along the sidewall [Figure6(a2)]. Afterward, the 13XMS beads reach the bottom corner, as Figure 6(a3). After about ten minutes, the bed reaches stable state. It is observed that the 13XMS beads move downward along the sidewall and migrate upward slowly thought the steel beads based on the mass conservation laws. According to Figure 2(VI), the bed of single steel beads cannot generate convection at this vibration condition. However, by adding a small number of light 13XMS beads, the bed is activated. The 13XMS beads near the sidewall play the role of lubrication.

When the $16 \mathrm{~mm}$ 13XMS beads layer was initially placed below the steel beads [Figure 6(b1)], the 13XMS beads first rise through the steal beads bed and reach the bed surface [Figure6(b2) (b4)]. Then these 13XMS beads move along the sidewall, and finally format a stable distribution same with Figure 6(a4).

Figure $6(\mathrm{a}) \sim(\mathrm{d})$ show the stable convection and separation at $f=20 \mathrm{~Hz}, 30 \mathrm{~Hz}, 40 \mathrm{~Hz}$ and $60 \mathrm{~Hz}$. The convection patterns of the binary component also are similar with as that of single 13XMS bead [Figure 2(I) (IV)]. By comparison, the convection strength is weak as a large number of steel beads are difficult to fluidize. 


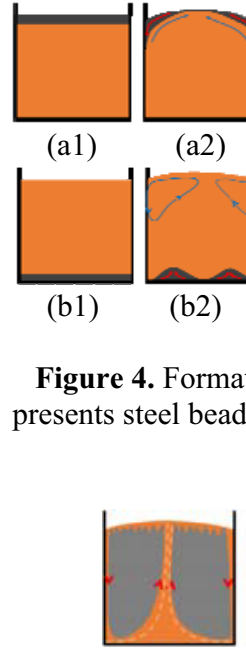

(a)

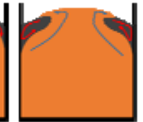

(a3)

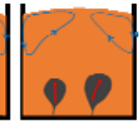

(b3)

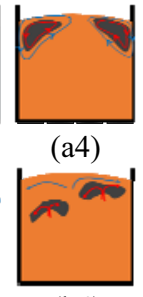

(b4)

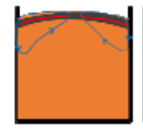

(b5)

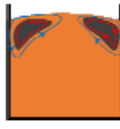

(b6)
Figure 4. Formation process of steel beads core. The dark $(10 \%$ volume ratio) and
$(90 \%$ volume ratio).

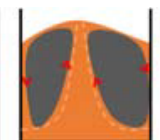

(b)

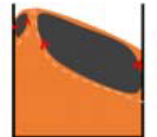

(c)

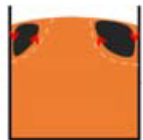

(d)
Figure 5. Separation of $13 \mathrm{XMS}$ and steel beads. The volume ratio of $13 \mathrm{XMS}$ and steel beads are $90 \%$ and $10 \%$, respectively.

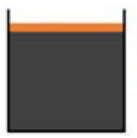

(a1)

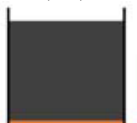

(b1)

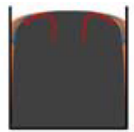

(a2)

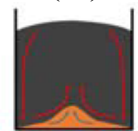

(b2)

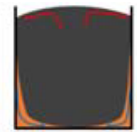

(a3)

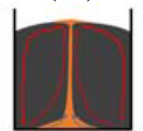

(b3)

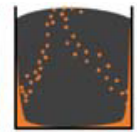

(a4)

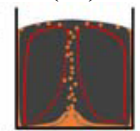

(b4)

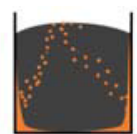

(b5)
Figure 6. Formation process of steel beads core. The dark presents steel beads ( $90 \%$ volume ratio) and the peak 13XMS ( $10 \%$ volume ratio).

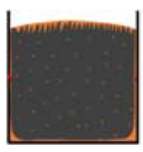

(a)

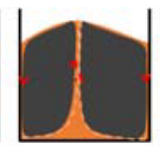

(b)

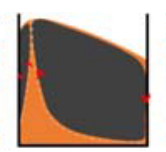

(c)

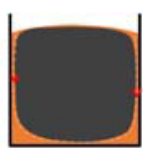

(d)
Figure 7. Separation of 13XMS and steel beads. The volume ratio of 13XMS and steel beads are $10 \%$ and $90 \%$, respectively.

\section{Conclusion}

By vertically vibrating single light particles (13XMS beads) in a narrow bed, the bed shows five different types patterns of granular motion, as global convection, symmetrical heap, unsymmetrical heap, local convection and pseudo solid state, by increasing the vibration frequency from $15 \mathrm{~Hz}$ to $70 \mathrm{~Hz}(\Gamma>3.0)$. The alteration between these patterns is mainly influenced by vibration frequency, and the acceleration is a minor factor. However, the heavy particles (steel beads) cannot be fluidized at these vibration condition and the bed only shows pseudo solid state.

When a small quantity of heavy particles $(10 \%$ volume ratio steel beads) are added into the 13XMS beads bed, the granular convection is hardly effected.
And the steel beads cluster in the center of the convection, as a core of convection roll. The initial distribution of binary particles will influence the aggregation process of steel beads, however, the final convection and separation are not influenced.

When $10 \%$ volume ratio $13 \mathrm{XMS}$ beads are added into the steel beads bed, the 13XMS will make the bed flowing. The bed changes from the pseudo solid to global convection, symmetrical heap, unsymmetrical heap or local convection, which is decided the convection of 13XMS. Comparing with the single $13 \mathrm{XMS}$, the binary granular bed has a weak convection strength.

\section{Acknowledgement}

The authors are grateful for support from the National Natural Science Foundation of China (Grants No. 51506006).

\section{References}

1. Y. H. Taguchi, Phys. Rev. Lett., 69, 1367 (1992)

2. M. A. Keiko, A. Tetsuo, Phys. Rev. Lett., 77, 4166 (1996)

3. A. D. Rosato, K. J. Strandburg, F. Prinz, R. H. Swendsen. Phys. Rev. Lett., 58, 1038(1987)

4. J. B. Knight, H. M. Jaeger, Phys. Rev. Lett., 70, 3728 (1993)

5. N. Burtally, P. J. King, M. R. Swift, Science, 295, 1877, (2002)

6. P. Biswas, P. Sánchez, M. R. Swift, P. J. King, Phys. Rev. E, 68, 050301 (2003)

7. Q. Shi, G. Sun, M. Hou, K. Lu, Phys. Rev. E, 75, 061302 (2007)

8. T. Akiyama, K. M. Aoki, K. Yamamoto, T. Yoshikawa, Granular Matter, 1, 15 (1998)

9. S. C. Yang, Powder Technol., 164, 65 (2006)

10. S. S. Hsiau, W. C. Chen, Adv. Powder Technol., 13, 301(2002)

11. C. H. Tai, S. S. Hsiau, C. A. Kruelle, Powder Technology, 204, 255 (2010)

12. D. Brone, F. J. Muzzio, Phys. Rev. E, 56, 1059 (1997).

13. E. E. Ehrichs, H. M. Jaeger, G. S. Karczmar, J. B. Knight, V. Y. Kuperman, S. R. Nagel, Nature, 267, 1632 (1995)

14. Y. S. Wong, C. H. Gan, C. H. Wang, X. Fan, D. J. Parker, A. Ingram, J. P. K. Seville, Phys. Fluids, 18, 043302 (2006)

15. M. Majid, P. Walzel, Powder Technol., 192, 311 (2009)

16. P. Eshuis, K. van der Weele, D. van der Meer, R. Bos, D. Lohse, Phys. Fluids, 19, 123301 (2007)

17. F. Zhang, L. Wang, C. Liu, P. Wu, S. Zhan, Phys. Lett. A, 378, 1303 (2014) 